\title{
The altitude of Neptune cloud features from high-spatial-resolution near-infrared spectra
}

\author{
S.G. Gibbard, ${ }^{\mathrm{a}, *}$ I. de Pater, ${ }^{\mathrm{b}}$ H.G. Roe, ${ }^{\mathrm{c}}$ S. Martin, ${ }^{\mathrm{b}}$ B.A. Macintosh,${ }^{\mathrm{a}}$ and C.E. Max ${ }^{\mathrm{a}, \mathrm{d}}$ \\ a Lawrence Livermore National Laboratory, Livermore, CA 94550, USA \\ ${ }^{\mathrm{b}}$ Astronomy Department, 601 Campbell Hall, University of California, Berkeley, CA 94720, USA \\ ${ }^{\mathrm{c}}$ Division of Geological and Planetary Sciences, California Institute of Technology, Pasadena, CA 91125, USA \\ ${ }^{\mathrm{d}}$ Center for Adaptive Optics, University of California at Santa Cruz, Santa Cruz, CA 94064, USA
}

Received 5 March 2003; revised 16 July 2003

\begin{abstract}
We report on observations of Neptune from the 10-meter W.M. Keck II Telescope on June 17-18 (UT) 2000 and August 2-3 (UT) 2002 using the adaptive optics (AO) system to obtain a spatial resolution of 0.06 arcseconds. With this spatial resolution we can obtain spectra of individual bright features on the disk of Neptune in a filter centered near 2 microns. The use of a gas-only, simple reflecting layer radiative transfer model allows us to estimate the best fit altitudes of 18 bright features seen on these 4 nights and to set a constraint on the fraction of hydrogen in ortho/para equilibrium. On these nights there were three main types of features observed: northern hemisphere features in the range from +30 to -45 degrees; southern hemisphere features in the range from -30 to -50 degrees; and small southern features at -70 degrees. We find that the altitudes of the northern features are in the range from $0.023-0.064$ bar, which places them in Neptune's stratosphere. Southern features at -30 to -50 degrees are mainly at altitudes from 0.10 to 0.14 bars. The small features at -70 degrees are somewhat deeper in the upper troposphere, at 0.17 and 0.27 bars. This pattern of features located at higher altitudes in the northern hemisphere and lower altitudes in the south has also been noted by previous observers. The best fits for all the observed spectra give a value of 1.0 for the fraction of hydrogen in ortho/para equilibrium; the value of the helium fraction is less well constrained by the data at 0.24 . We suggest that the southern mid-latitude features are methane haze circulated up from below, while the $-70^{\circ}$ features may be isolated areas of upwelling in a general area of subsidence. Northern bright features may be due to subsidence of stratospheric haze material rather than upwelling and condensation of methane gas. We suggest that convection efficiently transports methane ice clouds to the tropopause in the Southern mid latitudes and thus plays a key role in the stratospheric haze production cycle.

Published by Elsevier Inc.
\end{abstract}

Keywords: Neptune, atmosphere; Infrared observations

\section{Introduction}

The planet Neptune, when observed at near-infrared wavelengths, shows a striking pattern of bright features at mid-latitudes against a predominantly dark background. These bright features (often called 'clouds' or 'storms') dominate the light from Neptune at wavelengths near $2 \mu \mathrm{m}$. This is in contrast to the planet's much more uniform appearance in visible light. Relatively dark features seen at visible wavelengths are thought to originate in the planet's troposphere (Stratman et al., 2001), whereas the infraredbright clouds are believed to be located near the tropopause

\footnotetext{
* Corresponding author.

E-mail address: sgibbard@igpp.ucllnl.org (S.G. Gibbard).
}

and perhaps in the stratosphere (Baines et al., 1995a; Roe et al., 2001). At both visible and near-infrared wavelengths, the light we receive from Neptune is reflected sunlight. At blue wavelengths, the cross section for molecular Rayleigh scattering is large, thus the disk of the planet is bright with low contrast brighter and darker features. The Rayleigh scattering cross section decreases dramatically with increasing wavelength and thus is not an important radiative process at near-infrared wavelengths. Methane absorption and $\mathrm{H}_{2}$ collision-induced absorption (H2-CIA) become important radiative transfer processes at select visible and near infrared wavelength bands (see, for example, Baines et al., 1995a). Thus, in near infrared images taken through filters which span methane and H2-CIA bands (such as K band), the disk of Neptune appears dark, with distinct bright cloud features. 
By tracking the movement of Neptune's infrared-bright features, rotation rates at various latitudes can be determined (e.g., Hammel et al., 1989; Sromovsky et al., 1993, 1995; Gibbard et al., 2002). The time variation (on both short and long time scales) of cloud features gives information on the dynamics of Neptune's atmosphere (e.g., Martin et al., 2000, 2001, 2002; Sromovsky et al., 1995, 2001a, 2001b, 2001c, 2002). Also of interest is the vertical distribution of the haze: at which pressure level do the 'storms' occur, and how does this vary across the planet? Stratospheric haze layers extend from somewhat above the tropopause (at $\sim 0.1$ bar) up to perhaps the millibar level (Baines et al., 1995a). These layers are composed of hydrocarbons such as $\mathrm{C}_{2} \mathrm{H}_{2}$ and $\mathrm{C}_{2} \mathrm{H}_{6}$, produced via photolysis of methane gas followed by a series of chemical reactions and condensation (Romani and Atreya, 1988). These hazes can be probed when observing at infrared wavelengths that are sensitive to methane and hydrogen absorption. By obtaining spectra of Neptune at near-infrared wavelengths we therefore gain a picture of the vertical structure of Neptune's stratosphere.

In the mid-to-late nineties we used the NIRC speckle system at Keck to image Neptune at H-band $(1.6 \mu \mathrm{m})$. These images showed the planet to be overall quite dark due to absorption by methane gas, with prominent infrared-bright regions mainly in the southern hemisphere (Gibbard et al., 2002). In 1999-2000 we imaged the planet using adaptive optics (AO) techniques (Max et al., 2000, 2002), and we took conventional (i.e., $\sim 0.5^{\prime \prime}$ spatial resolution) spectra (with the near-infrared spectrometer NIRSPEC) during engineering commissioning time. Using a model of gas absorption in Neptune's atmosphere, we estimated the altitude of bright northern and southern cloud features (Roe et al., 2001). The northern feature was found to be at a pressure level of 0.084 bars, and the southern feature at 0.14 bars. An analysis by Sromovsky et al. (2002), using data from the Infrared Telescope Facility, puts the altitudes of a number of cloud features at between 0.05 and 0.23 bars, while observations by Roddier et al. (1998) suggested that the bright features are located near pressures of 0.1 bars (or possibly higher).

When, in 2000, the Keck Telescope's AO system was fully commissioned, we observed Neptune with the adaptive optics system coupled to NIRSPEC to acquire spatiallyresolved spectra. With the arrival of the new NIRC2 camera and spectrograph in 2002, we obtained additional $2 \mu \mathrm{m}$ ( $\mathrm{K}$ band) spectra at a spectral resolution $R=2000$ and a spatial resolution of 0.06 arcseconds. In this paper we present an analysis of our spectral data using the method of Roe et al. (2001). This analysis indicates that, while most of the southern hemisphere cloud features are located near the tropopause at pressures of 0.08-0.14 bars, some of the features in the northern hemisphere are located in the stratosphere, at pressure levels $\leqslant 0.05$ bars.

\section{Observations and data reduction}

We observed Neptune with the 10-m W.M. Keck II telescope $^{1}$ on Mauna Kea, Hawaii, on June 17-18 (UT) 2000 and August 2-3 (UT) 2002. The observations during 2000 were made using NIRSPEC, Keck's 1-5 $\mu \mathrm{m}$ near-infrared spectrometer (McClean et al., 2000), in its low-resolution ( $R=2000)$ mode. In 2002 we obtained spectra using the newly-commissioned NIRC2 camera and spectrograph, a $1024 \times 1024$ Aladdin-3 InSb array, ${ }^{2}$ at a similar $R=2000$. During these times Neptune's sub-Earth latitude (planetographic) was $\sim-28^{\circ}$, so that our images show primarily Neptune's southern hemisphere.

Table 1 summarizes dates, times, and wavelengths of the Neptune images used to position the spectrograph slits. NIRSPEC has a slit-viewing camera, SCAM (a $256 \times 256$ HgCdTe PICNIC array with $0.017^{\prime \prime}$ pixels), which was used to obtain images at the same time as the spectroscopic observations. These images are shown in Fig. 1, where the location of the slit appears as a dark line. In this case the slit width was $0.072^{\prime \prime}$, which corresponds to 4 pixels in the SCAM images. For NIRC2, images were obtained just before and after the spectra in order check the position of the spectrograph slit. NIRC2 has available fine, medium, and coarse plate scales with pixels of size $0.01,0.02$, and 0.04 arcseconds. Figure 2 shows 0.01 arcsecond pixel images of Neptune obtained on August 2-3, 2002. In Fig. 3 images are shown with pixels of 0.04 arcseconds, the same platescale used for the spectrograph slit.

Table 2 summarizes the spectra of Neptune obtained, as well as the slit widths and exposure times. Wavelengths of

Table 1

Images used to position spectrograph slit

\begin{tabular}{lccccr}
\hline Date & $\begin{array}{c}\text { Time } \\
\text { (UTC) }\end{array}$ & Filter & $\begin{array}{c}\text { Platescale } \\
(\operatorname{arcsec})\end{array}$ & Airmass & $\begin{array}{r}T_{\text {exp }} \\
(\mathrm{sec})\end{array}$ \\
\hline June 17, 2000 & $11: 15$ & $\mathrm{~N} 6$ & 0.017 & 1.35 & 60 \\
& $11: 45$ & $\mathrm{~N} 6$ & 0.017 & 1.38 & 60 \\
June 18, 2000 & $11: 40$ & $\mathrm{~N} 6$ & 0.017 & 1.39 & 60 \\
August 2, 2002 & $11: 05$ & $\mathrm{~K}^{\prime}$ & 0.01 & 1.28 & 60 \\
& $11: 11$ & $\mathrm{~K}^{\prime}$ & 0.04 & 1.28 & 5 \\
& $11: 35$ & $\mathrm{~K}^{\prime}$ & 0.04 & 1.33 & 5 \\
& $11: 55$ & $\mathrm{~K}^{\prime}$ & 0.04 & 1.37 & 5 \\
August 3, 2002 & $12: 19$ & $\mathrm{~K}^{\prime}$ & 0.04 & 1.46 & 5 \\
& $11: 06$ & $\mathrm{Br}^{\prime}$ & 0.04 & 1.28 & 30 \\
& $11: 29$ & $\mathrm{~K}^{\prime}$ & 0.04 & 1.33 & 5 \\
& $11: 58$ & $\mathrm{~K}^{\prime}$ & 0.04 & 1.39 & 5 \\
& $12: 24$ & $\mathrm{~K}^{\prime}$ & 0.04 & 1.50 & 5 \\
& $12: 47$ & $\mathrm{H}$ & 0.01 & 1.61 & 60 \\
\hline
\end{tabular}

\footnotetext{
1 The Keck telescope is jointly owned and operated by the University of California and the California Institute of Technology.

2 Designed by Keith Matthews and Tom Soifer, both of Caltech. The instrument was built by Keith Matthews and engineer Sean Lin of Caltech, with help from James Larkin, Ian McLean, and others at UCLA (detector electronics and related software), and Al Conrad, Bob Goodrich, and Allan Honey at Keck Observatory (software). Support in Waimea was provided by Jim Bell, Randy Campbell, and Drew Medeiros.
} 

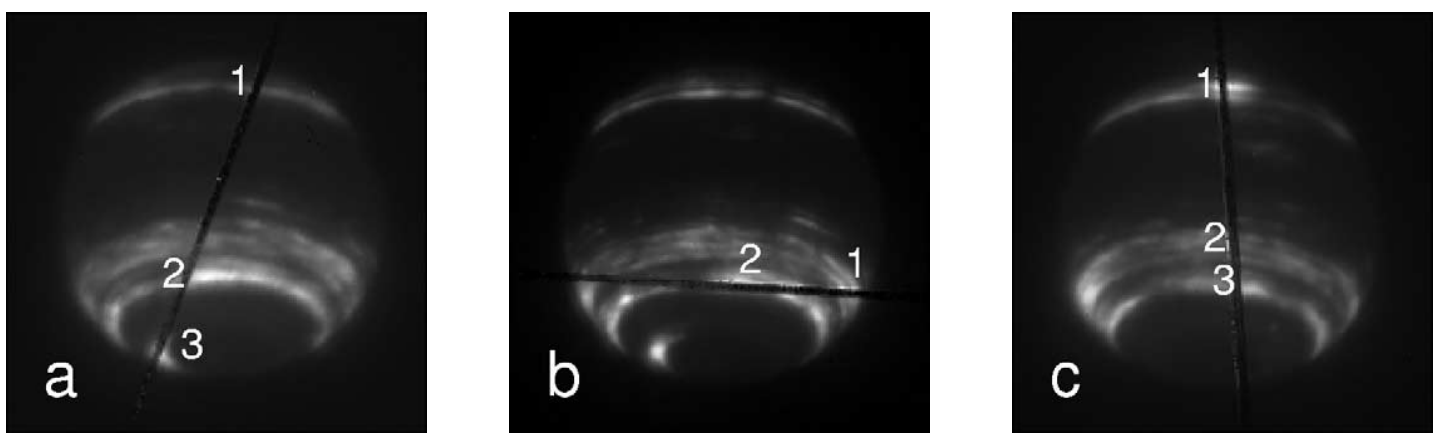

Fig. 1. Images of Neptune taken by SCAM, the NIRSPEC imaging camera, on June 17-18, 2000. The dark line shows the location of the spectrograph slit, and the locations of extracted spectra are numbered (the numbers correspond to those given in Table 4). The plate scale is $0.017^{\prime \prime}$ pixel ${ }^{-1}$, the sub-Earth latitude is $-28^{\circ}$, and north is up. (a) N6 image from June 17, 2000, 11:15. (b) N6 image from June 17, 2000, 11:45. (c) N6 image from June 18, $2000,11: 40$.
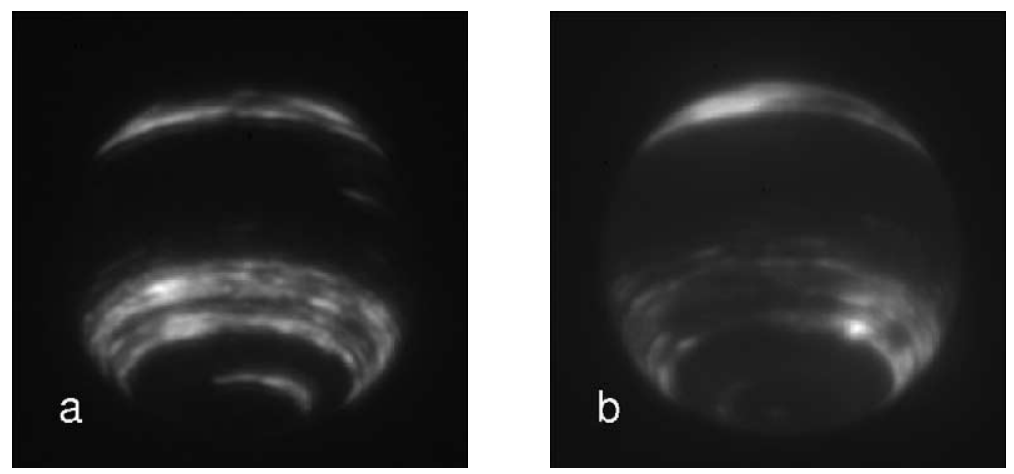

Fig. 2. Images of Neptune taken by NIRC2 on August 2 (a) and 3 (b), 2002, using the narrowfield camera (pixels are $\left.0.01^{\prime \prime}\right)$. The filter was H (1.48-1.78 um).

the filters used are given in Table $3 .{ }^{3}$ Reduction of the spectroscopic data consisted of sky and bias subtraction, rectification (for the NIRSPEC images, where the spatial and spectral coordinates are distorted with respect to the pixels on the array), and wavelength calibration. Wavelength calibration of the NIRSPEC images was by means of argon line lamps (calibration and rectification of NIRSPEC spectra is described in detail in Roe et al., 2001), while the NIRC2 data were calibrated based on $\mathrm{OH}$ lines from the Earth's atmosphere (Rousselot et al., 2000). In order to correct for telluric absorption, we observed A-type stars (shown in Table 2) which were divided by the spectrum of Vega (spectral type A0 V; Colina et al., 1996b) to get an estimate of the atmospheric and instrumental transfer function. The Neptune spectra were then divided by this transfer function.

For the NIRSPEC data on June 17 UT, pairs of spectra were averaged in order to improve the signal-to-noise in the spectra. (Due to rotation and changes in features on Neptune we combined only images taken within a $\sim 10$ minute interval, which corresponds to a rotation of $\simeq 3.5^{\circ}$ or about 2.5 pixels.) A single spectrum was analyzed on June 18. For the NIRSPEC June 17 and 18 data, the spatial resolution of the spectra obtained was $0.06^{\prime \prime}$, based on the FWHM of stellar spectra. This corresponds to about 6 pixels in the spatial

\footnotetext{
3 Filter traces can be found at the Keck Observatory website, http://www2.keck.hawaii.edu:3636/.
}

Table 2

Spectra

\begin{tabular}{lclllr}
\hline Date & $\begin{array}{c}\text { Time } \\
\text { (UTC) }\end{array}$ & Filter & $\begin{array}{c}\text { Slit width } \\
(\operatorname{arcsec})\end{array}$ & Airmass & $\begin{array}{r}T_{\text {exp }} \\
(\mathrm{sec})\end{array}$ \\
\hline June 17, 2000 & & & & & \\
$\quad$ Neptune & $11: 17$ & $\mathrm{~N} 6$ & 0.072 & 1.35 & 300 \\
$\quad$ Neptune & $11: 24$ & $\mathrm{~N} 6$ & 0.072 & 1.36 & 300 \\
Neptune & $12: 08$ & $\mathrm{~N} 6$ & 0.072 & 1.38 & 300 \\
Neptune & $12: 15$ & $\mathrm{~N} 6$ & 0.072 & 1.39 & 300 \\
HD201941, A2 & $12: 32$ & $\mathrm{~N} 6$ & 0.072 & 1.30 & 30 \\
June 18, 2000 & & & & & \\
Neptune & $11: 49$ & $\mathrm{~N} 6$ & 0.072 & 1.39 & 300 \\
HD210941, A2 & $11: 30$ & $\mathrm{~N} 6$ & 0.072 & 1.26 & 30 \\
August 2, 2002 & & & & & \\
$\quad$ Neptune & $11: 19$ & $\mathrm{~K}^{\prime}$ & 0.040 & 1.29 & 300 \\
Neptune & $11: 27$ & $\mathrm{~K}^{\prime}$ & 0.040 & 1.31 & 300 \\
Neptune & $12: 03$ & $\mathrm{~K}^{\prime}$ & 0.040 & 1.42 & 300 \\
Neptune & $12: 09$ & $\mathrm{~K}^{\prime}$ & 0.040 & 1.44 & 300 \\
HIP102702, A0 & $11: 42$ & $\mathrm{~K}^{\prime}$ & 0.040 & 1.39 & 30 \\
August 3, 2002 & & & & & \\
Neptune & $11: 16$ & $\mathrm{~K}^{\prime}$ & 0.040 & 1.30 & 300 \\
Neptune & $11: 23$ & $\mathrm{~K}^{\prime}$ & 0.040 & 1.31 & 300 \\
Neptune & $12: 34$ & $\mathrm{~K}^{\prime}$ & 0.040 & 1.54 & 300 \\
Neptune & $12: 40$ & $\mathrm{~K}^{\prime}$ & 0.040 & 1.59 & 300 \\
HIP102702, A0 & $11: 40$ & $\mathrm{~K}^{\prime}$ & 0.040 & 1.41 & 30 \\
\hline
\end{tabular}

dimension on NIRSPEC. The NIRSPEC spectra were thus spatially averaged over 6 pixels (using an optimal weighted extraction technique to mitigate the effect of bad pixels and cosmic ray hits) to obtain spectra of individual features. These spectra are shown in Fig. 4. The NIRC2 data were 

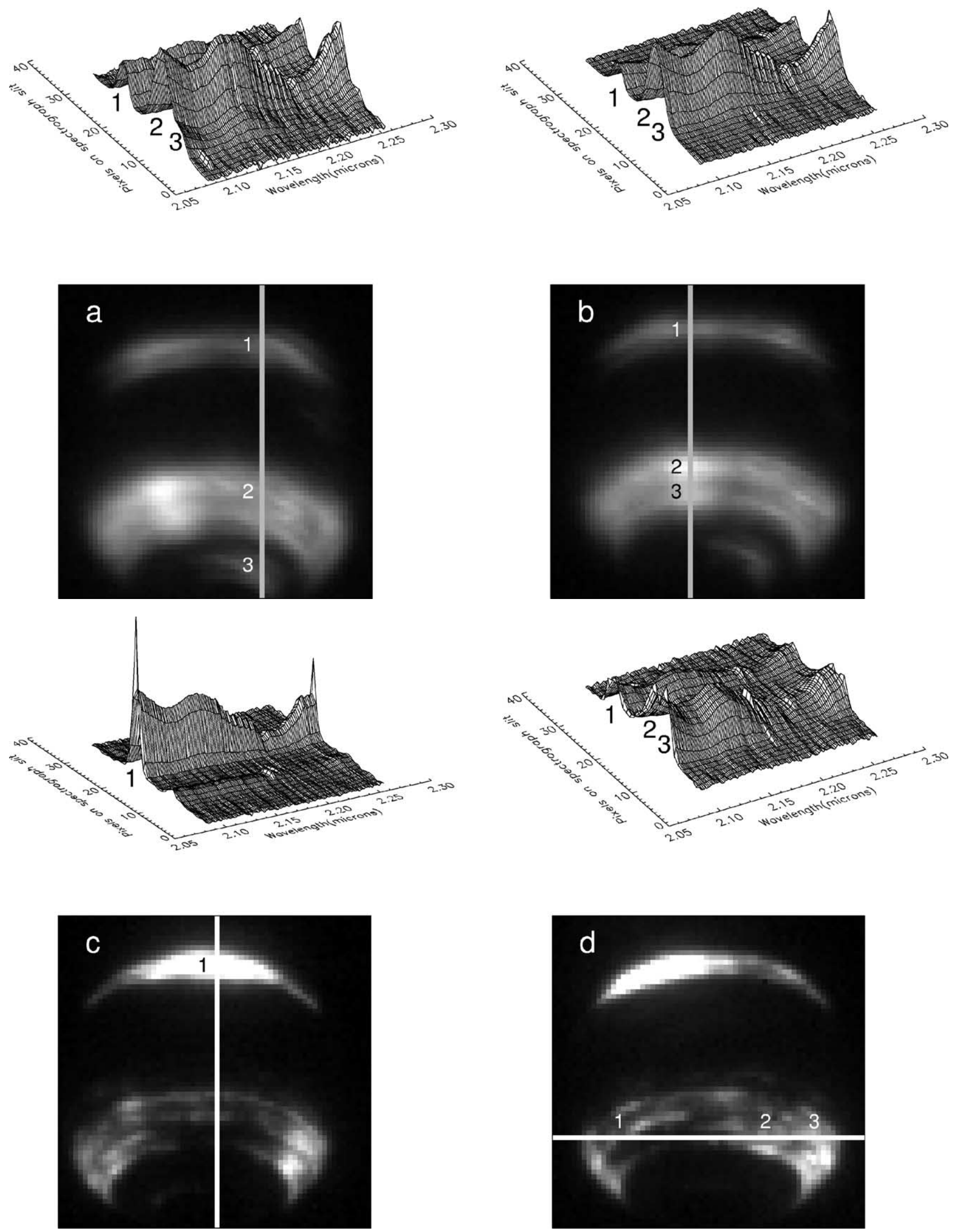

Fig. 3. Images and spectra of Neptune taken by NIRC2 on August $2-3,2002$. The plate scale is $0.04^{\prime \prime}$, and the colored lines show the location of the spectrograph slit. The locations of extracted spectra are numbered (the numbers correspond to those given in Table 4 and Fig. 4). Top panels show the spectra associated with the slit in the images shown below them; the locations of the extracted spectra are indicated. (a) $\mathrm{K}^{\prime}$ image from August 2 , 11:11. (b) $\mathrm{K}^{\prime}$ image from August 2, 11:35. (c) $\mathrm{K}^{\prime}$ image from August 3, 11:29. (d) $\mathrm{K}^{\prime}$ image from August 3, 12:24. 
Table 3

Filter characteristics

\begin{tabular}{lcc}
\hline Filter name & Central wavelength $(\mu \mathrm{m})$ & Wavelength range $(\mu \mathrm{m})$ \\
\hline $\mathrm{K}^{\prime}$ (NIRC2) & 2.12 & $1.95-2.30$ \\
$\mathrm{H}$ (NIRC2) & 1.63 & $1.48-1.78$ \\
$\mathrm{Br} \gamma$ (NIRC2) & 2.1643 & $2.1478-2.1807$ \\
N6 (NIRSPEC) & 1.94 & $1.558-2.315$ \\
\hline
\end{tabular}

Table 4

Altitudes of disk features

\begin{tabular}{clrcl}
\hline Date and time (UT) & Feature & $\begin{array}{c}\text { Latitude } \\
\left({ }^{\circ}\right)\end{array}$ & $\begin{array}{c}\text { Longitude } \\
\left({ }^{\circ}\right)\end{array}$ & $\begin{array}{l}\text { Press III) } \\
(\text { bars })\end{array}$ \\
\hline June 17, 2000 & 1. N. feature & 30 & 165 & 0.064 \\
$(11: 17+11: 24)$ & 2. S. feature & -35 & 135 & 0.11 \\
& 3. S. pole feature & -70 & 75 & 0.17 \\
June 17, 2000 & 1. Right limb & -40 & 235 & 0.11 \\
$(12: 08+12: 15)$ & 2. Center disk & -50 & 175 & 0.14 \\
June 18, 2000, 11:49 & 1. N. feature & 35 & 335 & 0.064 \\
& 2. S. feature & -35 & 355 & 0.144 \\
August 2, 2002, 11:19 & 3. 2nd S. feature & -50 & 0 & 0.21 \\
& 1. N. feature & 45 & 200 & 0.023 \\
2. S. feature & -35 & 200 & 0.11 \\
August 2, 2002, 12:03 & 3. S. pole feature & -70 & 200 & 0.27 \\
& 1. N. feature & 35 & 165 & 0.058 \\
2. S. feature & -35 & 165 & 0.10 \\
August 3, 2002, 11:16 & 3. 2nd S. feature & -45 & 165 & 0.12 \\
August 3, N. feature & 40 & 345 & 0.044 \\
& 1. Left feature & -30 & 320 & 0.13 \\
& 2. Right feature & -45 & 60 & 0.12 \\
& 3. Right limb & -30 & 85 & 0.13 \\
\hline
\end{tabular}

averaged over 3 pixels (a spatial scale of $0.12^{\prime \prime}$ ). The NIRC2 spectra are shown in Fig. 5. The wavelength range covered per pixel is $3.354 \times 10^{-4}$ microns. Based on observations of telluric $\mathrm{OH}$ lines, the spectral resolution achieved for the NIRC2 spectra was 3 pixels, which gives $R=\frac{\delta \lambda}{\lambda}=2100$. Table 4 gives a list of the features analyzed and their approximate latitudes; these features are individually numbered in Figs. 4 and 5. Features were chosen to be well separated in the images so that contamination of light from other features would not occur.

\section{Neptune model atmosphere}

In order to estimate the vertical location of the bright features in our images, we follow the procedure used by Roe et al. (2001): we construct a model gas-only atmosphere which gives the atmospheric absorption down to a given pressure level. The temperature profile used is that of Lindal (1992). In the range of $2.0-2.3 \mu \mathrm{m}$, the primary opacity is due to $\mathrm{H}_{2}$ collision-induced absorption and methane absorption. This opacity varies considerably as a function of wavelength, making the relative flux in this wavelength region a good probe of vertical location in Neptune's atmosphere. The bright features are modeled as single, flat, reflecting layers at a given pressure level (the actual reflectivity of the layer does not matter since we are only modeling the shape of the spectrum and not the absolute intensity). The model
Table 5

Model parameter grid points

\begin{tabular}{ll}
\hline Parameter & Values in model grid \\
\hline $\mathrm{f}_{\mathrm{He}}$ & $0.09,0.12,0.15,0.18,0.21,0.24$ \\
$\mathrm{f}_{\mathrm{CH}_{4}, \mathrm{~s}}$ & $8.0 \times 10^{-5}, 3.5 \times 10^{-4}, 1.05 \times 10^{-4}, 1.7 \times 10^{-3} \mathrm{a}$ \\
$\mathrm{f}_{\mathrm{CH}_{4}, \mathrm{t}}$ & $0.022^{\mathrm{b}}$ \\
$\mathrm{f}_{\mathrm{eq}}$ & $0.0,0.5,0.6,0.7,0.8,0.9,1.0$ \\
Pressure & 120 layers evenly spaced in $\log P$ from 5.0 to $10^{-4}$ bars \\
\hline \multicolumn{1}{c}{ a This is the upper limit found by Baines and Hammel (1994). } \\
b Value from Baines et al. (1995b); our model is not sensitive to this.
\end{tabular}

spans the spectral region from 2.08-2.25 microns, avoiding a telluric $\mathrm{CO}_{2}$ band below $2.08 \mu \mathrm{m}$ and sharp methane features at $>2.25 \mu \mathrm{m}$ which are not well-represented by the methane coefficients in the model.

In order to model the $\mathrm{H}_{2}$ collision-induced absorption we use the routines of A. Borysow (Borysow, 1991; Zheng and Borysow, 1995; Borysow and Frommhold, 1989; Borysow et al., 1989). This absorption depends on the fraction of $\mathrm{H}_{2}$ in ortho/para equilibrium as well as the abundance of He. Neptune's $2 \mu \mathrm{m}$ spectrum is also strongly influenced by methane absorption, which is difficult to calculate in the near-IR due to the large number of methane lines at various strengths in this part of the spectrum. We have therefore used a correlated $k$-coefficient model as described by Lacis and Oinas (1991) and Goody and Yung (1989), using the methane coefficients of Irwin et al. (1996). Because the spectral resolution of this model was $5 \mathrm{~cm}^{-1}$, we are limited in the spectral resolution of the model spectra we construct. For this reason, we rebinned our NIRSPEC and NIRC2 spectra to the model resolution of $5 \mathrm{~cm}^{-1}$, which gives a total of 74 spectral data points over the range from 2.08-2.25 $\mu \mathrm{m}$. The spectral resolution of the rebinned spectra over this range is thus $R \sim 1000$.

For each feature, the airmass was calculated from the deprojected feature longitude and latitudes (since we averaged the spectra over several pixels, the average airmass was used). The airmass, along with the values of $\mathrm{f}_{\mathrm{He}}$ (the mole fraction of helium in the atmosphere), $\mathrm{f}_{\mathrm{CH}_{4}, \mathrm{~s}}$ and $\mathrm{f}_{\mathrm{CH}_{4}, \mathrm{t}}$ (the mole fraction of methane in stratosphere and troposphere), and $\mathrm{f}_{\mathrm{eq}}$ (the fraction of $\mathrm{H}_{2}$ in ortho/para equilibrium), was used to produce a grid of model spectra for features at various pressure levels in the atmosphere. The values considered in the grid are shown in Table 5. The predicted transmission spectrum was multiplied by the solar spectrum of Colina et al. (1996a) to predict the reflectance observed from the cloud features. The resulting model spectrum that best fit the data was then determined by minimizing the difference between the observed spectrum and the various models. Further details of this procedure are given in Roe et al. (2001).

\section{Results from modeling}

\subsection{NIRSPEC data}

Figure 6 shows the best fits to the features observed on June 17 and 18, 2000. The NIRSPEC data from June 17 have 

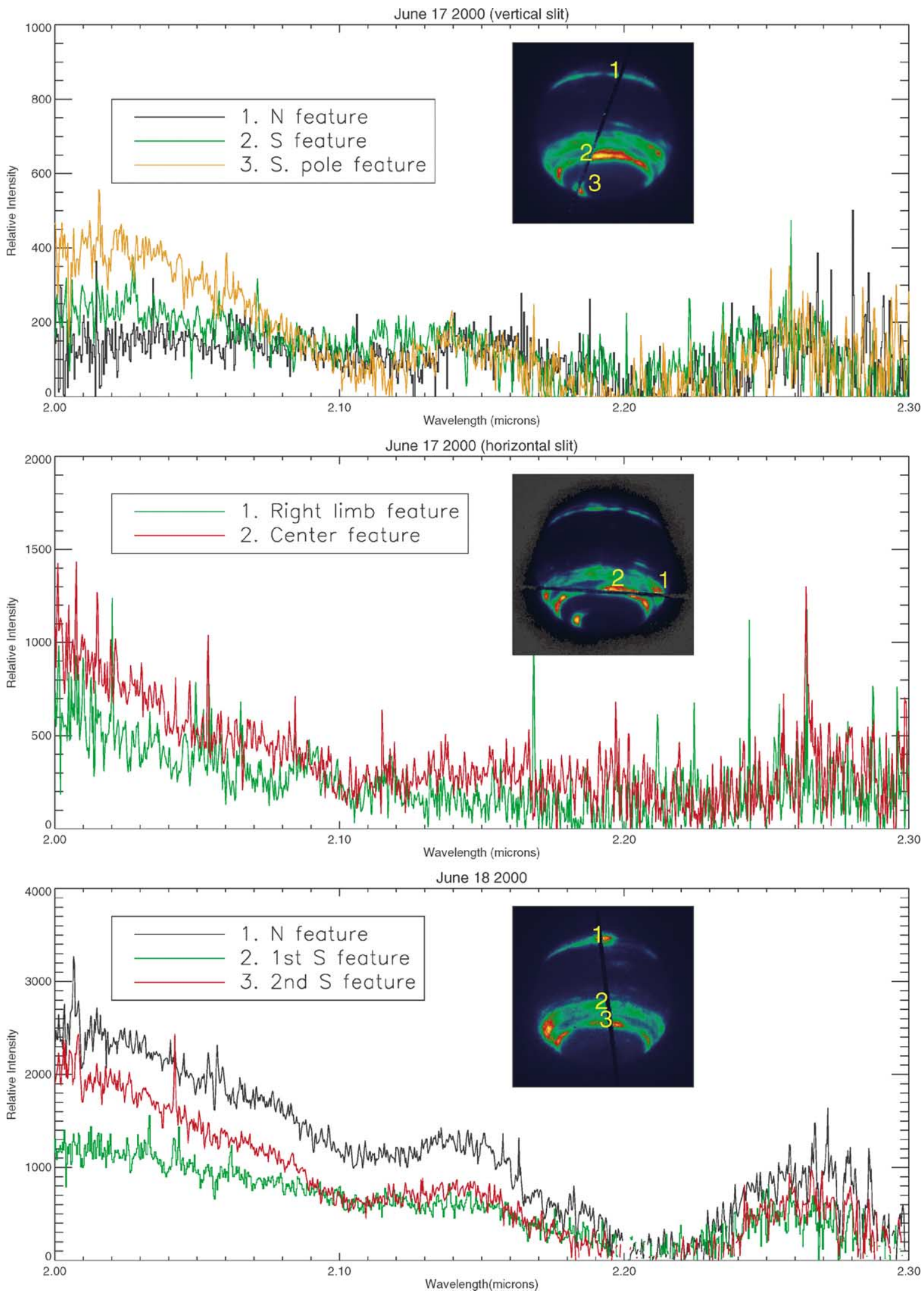

Fig. 4. Extracted spectra from NIRSPEC data on July 17 and 18, 2000. Spectra were extracted by averaging over 6 pixels ( 0.06 arcseconds or about 1250 km on Neptune). Note that the features observed on June 17 have a relatively low brightness, and therefore the signal/noise ratio is considerably lower than for the June 18 data (where the opposite side of the planet was observed). 

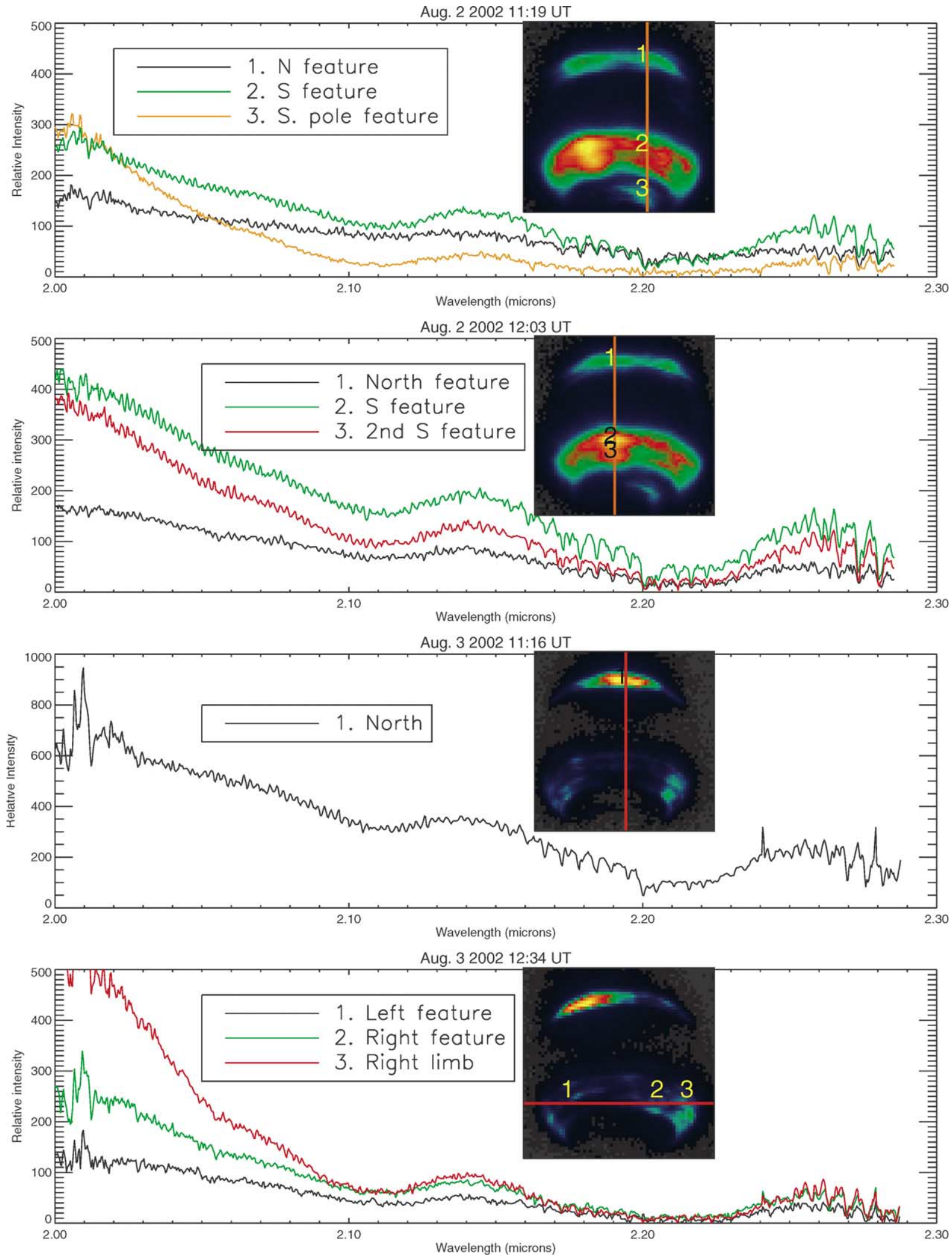

Fig. 5. Extracted spectra from NIRC2 data on August 2 and 3, 2002. Spectra were extracted by averaging over 3 pixels $(0.12$ arcseconds or about $2500 \mathrm{~km}$ on Neptune). 

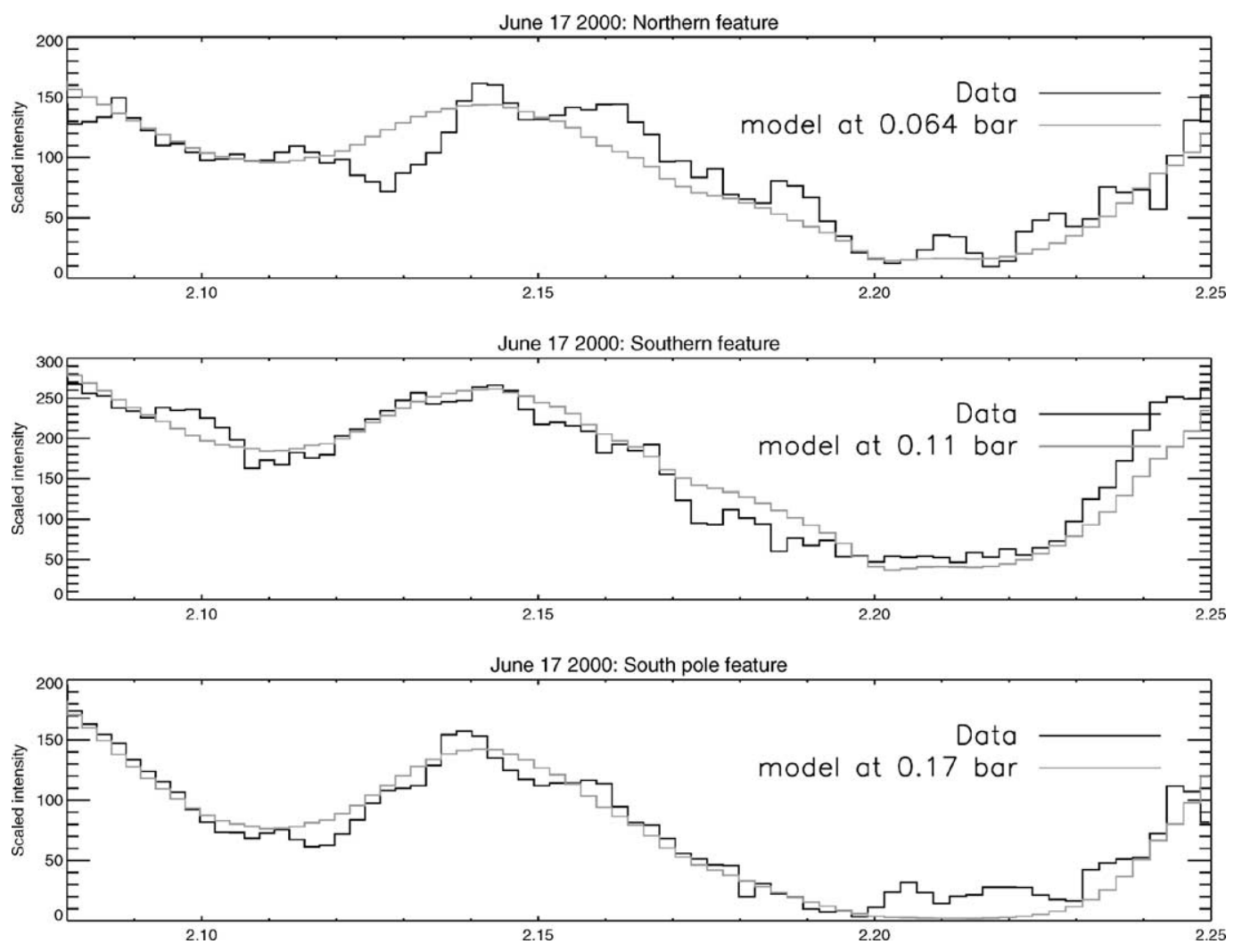

(a)
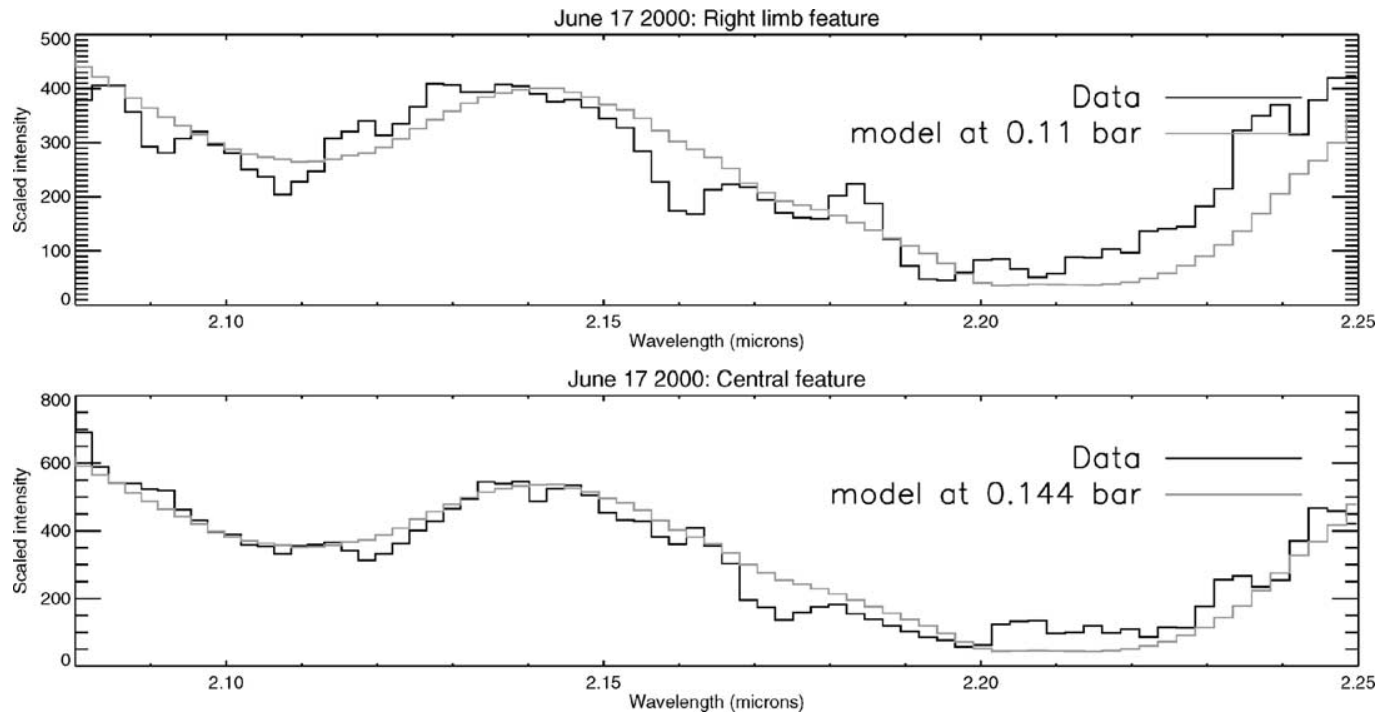

(b)

Fig. 6. Spectra and models from NIRSPEC data. All fits use the best fit parameters: $\mathrm{f}_{\mathrm{eq}}=1.0, \mathrm{f}_{\mathrm{He}}=0.24, \mathrm{f}_{\mathrm{CH}_{4}, \mathrm{t}}=0.022, \mathrm{f}_{\mathrm{CH}_{4}, \mathrm{~s}}=1.7 \times 10^{-3}$. The dark line shows the data, while the lighter line is the best fit model. (a) June 17, 2000, 11:17. (b) June 17, 2000, 12:08. (c) June 18, 2000, 11:49.

a lower signal/noise ratio than the June 18 data, mainly because the features observed on June 17 were fainter. June 18 sampled the opposite side of the planet (due to Neptune's rotation period of about 16 hours). Since we observed both sides of Neptune on these two nights, we have a fairly complete picture of the planet's activity at this time. The brightest feature present was the northern feature on June 18; there were also bright latitude bands at -35 and $-50^{\circ}$ and a small southern feature at $-70^{\circ}$ seen on June 17 . The best fit altitudes for these features are given in Table 4 . Our best fit models find that the features at 30-35 degrees north have an altitude of 0.064 bars (which is the same both for the bright feature on June 18 and for the dimmer 'background' haze on June 17). Bright features at -35 and -50 degrees have altitudes ranging from $0.11-0.21$ bars, while the small feature at $-70^{\circ}$ has a best fit altitude of 0.17 bars. We note that the 

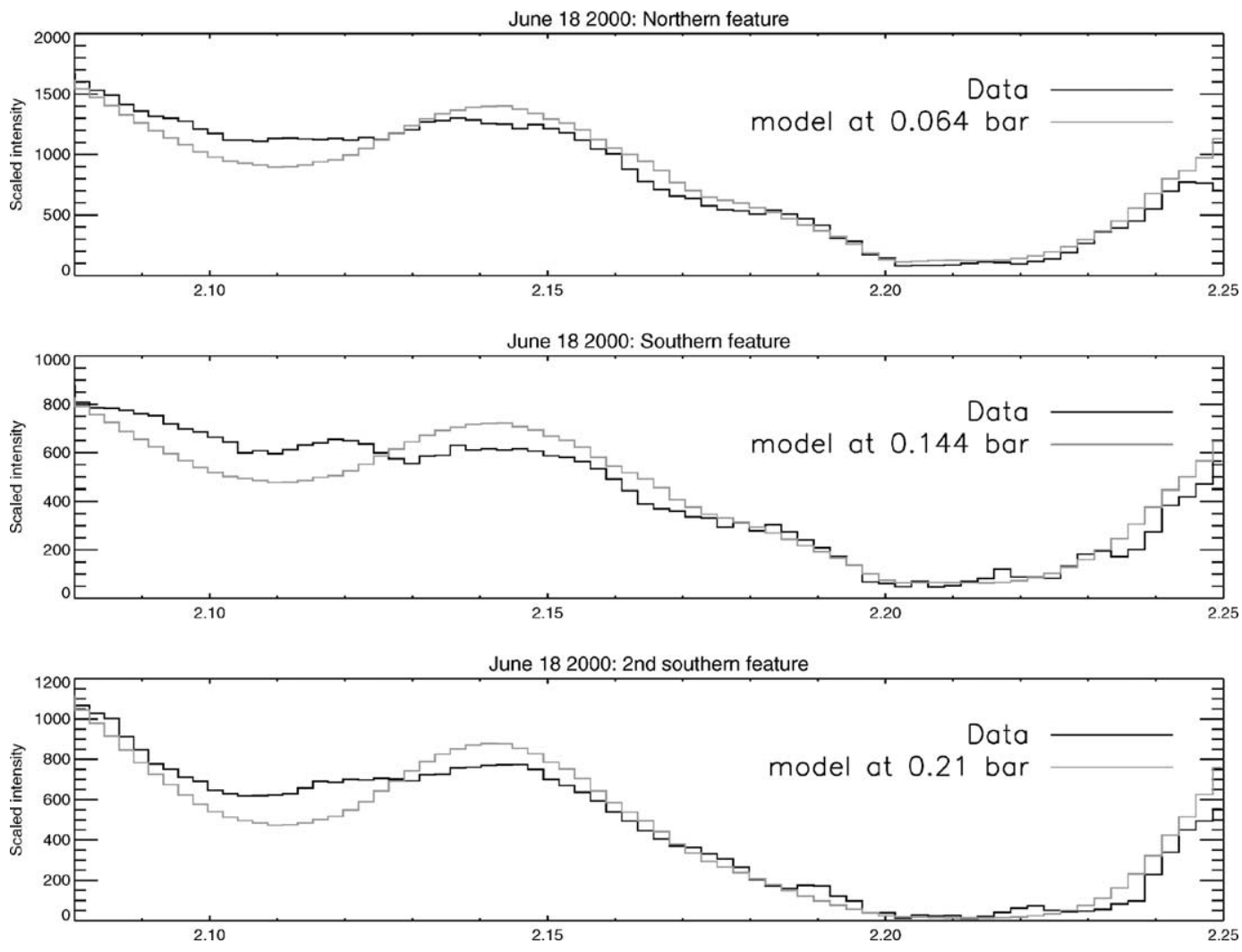

(c)

Fig. 6. Continued.

model fits for June 18 are relatively poor in the range from 2.10-2.15 microns; the flatness of the spectra in this region is not well fit by models that fit the data in the rest of the spectral range. In all cases for the 2000 data the best fit parameters were $\mathrm{f}_{\mathrm{eq}}=1.0$ and $\mathrm{f}_{\mathrm{He}}=0.24$ (although the models are much more sensitive to the $\mathrm{f}_{\mathrm{eq}}$ than the $\mathrm{f}_{\mathrm{He}}$; see below).

\subsection{NIRC2 data}

Figure 7 shows the best fits for August 2 and 3,2002. We were able to observe both sides of Neptune in observations 24 hours apart (about $1 \frac{1}{2}$ Neptune rotations). The brightest feature present was in the northern hemisphere and was observed on August 3. In the southern hemisphere there were bright bands at $-30^{\circ}$ to $-45^{\circ}$, with a single small bright feature at $-70^{\circ}$. Thus the overall appearance of Neptune in 2000 and 2002 was quite similar. The best fit altitude for the bright northern feature seen on August 3 was 0.044 bars, while the best fits for the 'background' northern haze seen on August 2 were altitudes of 0.023 and 0.058 bars. The southern features were located at $0.10-0.13$ bars, while the south pole feature was located deeper at 0.27 bars (this was the deepest feature identified in the dataset). As was the case for the 2000 data, the best fits were achieved with values of $\mathrm{f}_{\mathrm{eq}}=$ 1.0 and $\mathrm{f}_{\mathrm{He}}=0.24$. The model was insensitive to the value of $\mathrm{f}_{\mathrm{CH}_{4}, \mathrm{t}}$ and relatively insensitive to the value of $\mathrm{f}_{\mathrm{CH}_{4}, \mathrm{~s}}$; the values used here were 0.022 and $1.7 \times 10^{-3}$, respectively.
To give the reader a sense of the sensitivity to changes in the model altitude, Fig. 8 shows the effect of changing the altitude of the right feature (feature \#2) observed in the second spectrum from August 3, 2002. Changing the altitude from the best-fit value of 0.12 bar to values of 0.08 or 0.16 bar leads to clearly worse fits than the nominal value.

Figure 9 shows the sensitivity of the model to changes in $\mathrm{f}_{\mathrm{He}}$. The data shown are again for the right feature in the 2nd spectrum from August 3, 2002 (results are similar for the other spectra). Changing the value of $\mathrm{f}_{\mathrm{He}}$ to 0.09 or 0.18 gives a best fit of 0.10 or 0.11 bars compared to the nominal 0.12 for the best fit value of $\mathrm{f}_{\mathrm{He}}=0.24$. Thus the estimated altitude of the features does not depend sensitively on this parameter; by the same token we cannot constrain this parameter significantly.

Figure 10 shows the model sensitivity to changes in $\mathrm{f}_{\mathrm{eq}}$, the fraction of hydrogen in ortho/para equilibrium. Once again the data shown are from the right feature on the 2 nd spectrum from August 3,2002. In this case changing the parameter from its best fit value of 1.0 leads to a significant deterioration in the fit. The best fit altitude for $\mathrm{f}_{\text {eq }}=0.5$ is 0.16 bar, while for $\mathrm{f}_{\mathrm{eq}}=0.9$ it is 0.13 bar. It is clear, however, that the goodness of the model fit depends on a value of $\mathrm{f}_{\text {eq }} \simeq 1$.

Another parameter that is important in the model is the airmass of Neptune's atmosphere, which we parameterize as the two-way airmass including the photon path through 

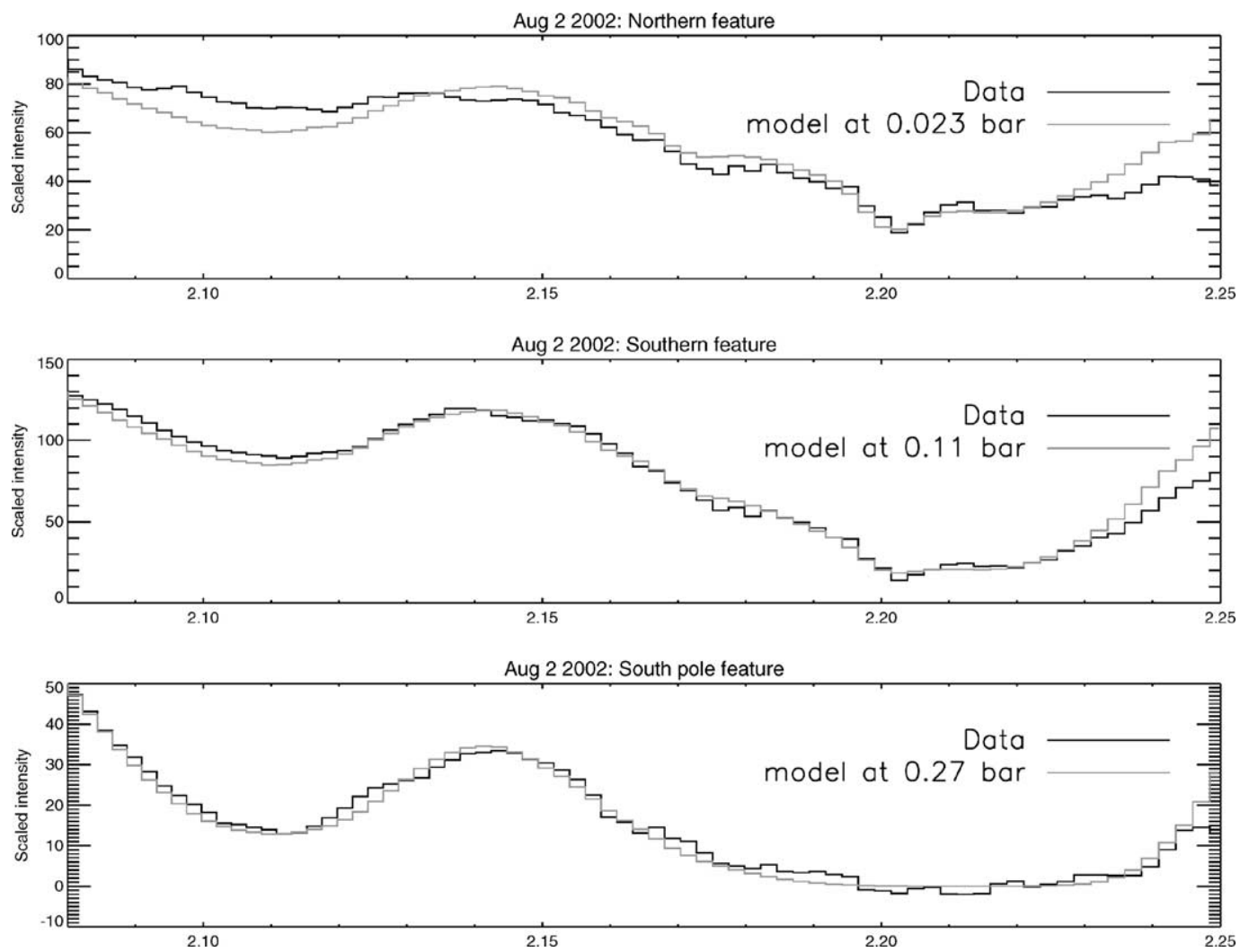

(a)
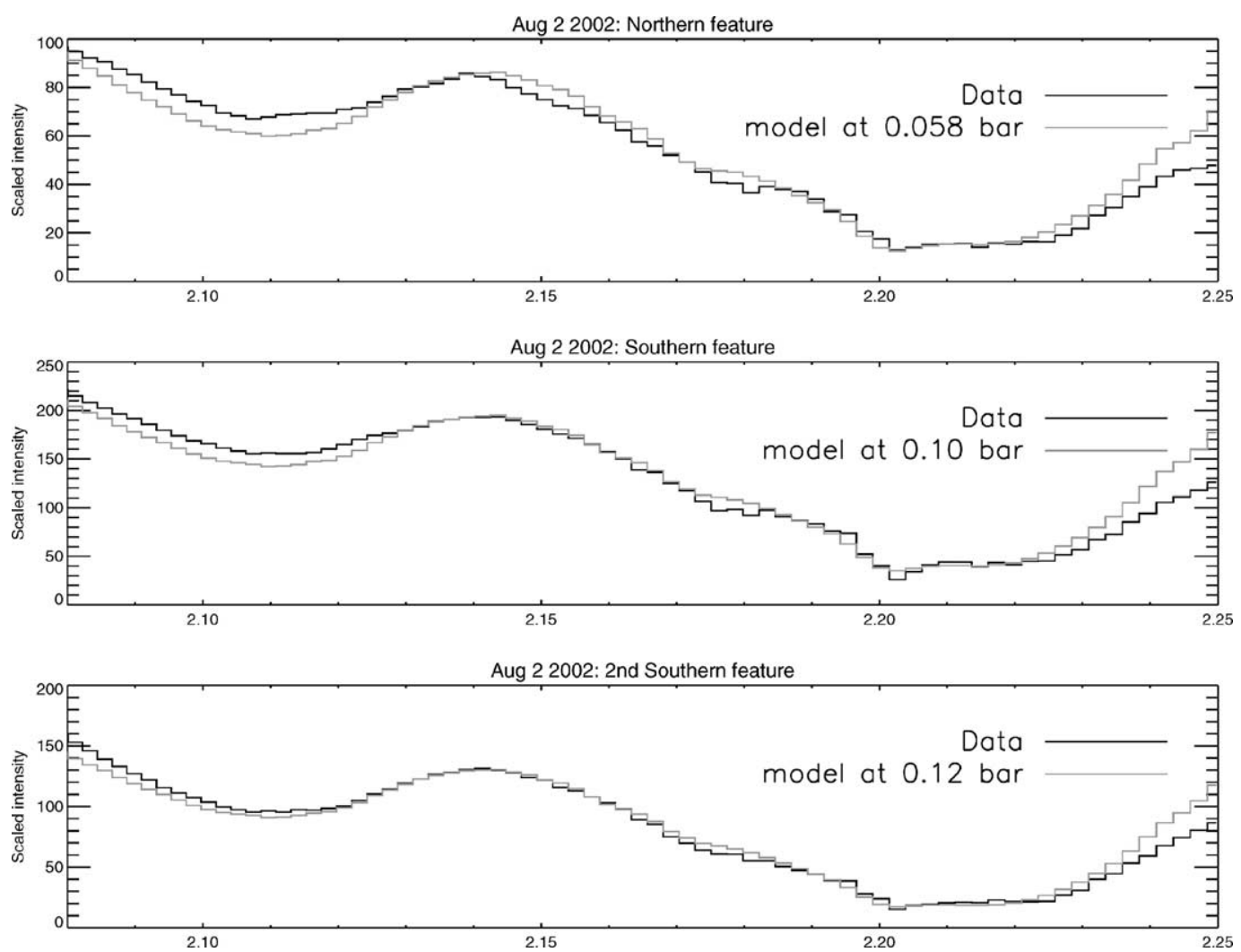

(b)

Fig. 7. Spectra and models from NIRC2 data. All fits use the best fit parameters: $\mathrm{f}_{\mathrm{eq}}=1.0, \mathrm{f}_{\mathrm{He}}=0.24, \mathrm{f}_{\mathrm{CH}_{4}, \mathrm{t}}=0.022, \mathrm{f}_{\mathrm{CH}_{4}, \mathrm{~s}}=1.7 \times 10^{-3}$. The dark line shows the data, while the lighter line is the best fit model. (a) August 2, 2002, 11:19. (b) August 2, 2002, 12:03. (c) August 3, 2002, 11:16. (d) August 3, 2002, 12:34. 


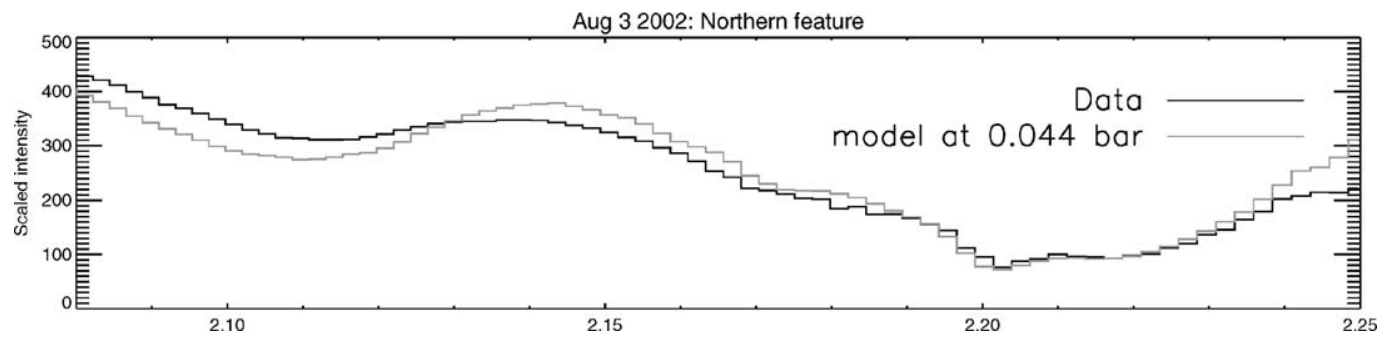

(c)
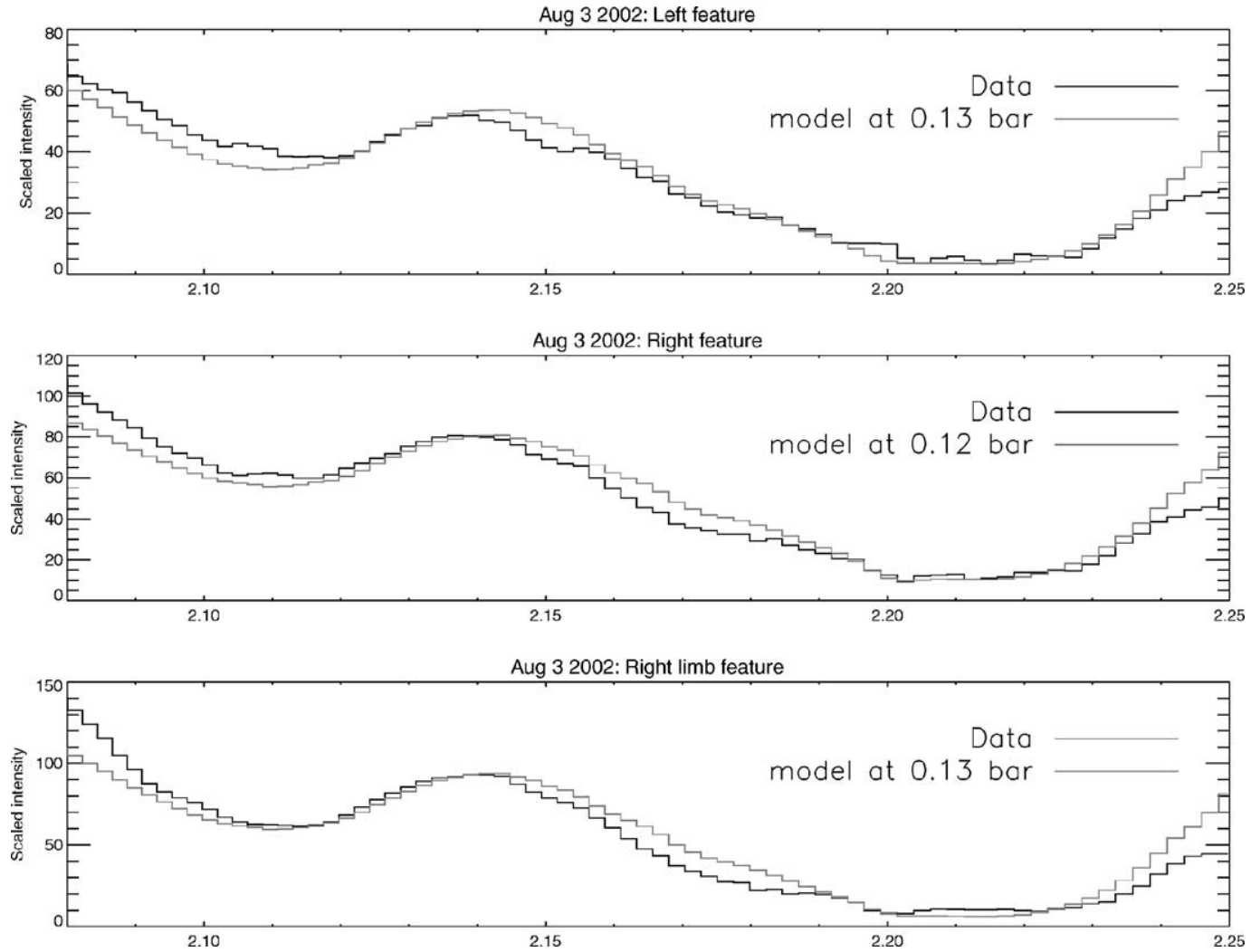

(d)

Fig. 7. Continued.

the atmosphere and back. This is determined by the latitude and longitude of the feature. Latitudes and longitudes of all features were determined by deprojection onto a latitudelongitude grid. For features near the center of the disk, where the two-way airmass is close to 2 and varies slowly, a small error in the determination of the airmass is not important, but for features close to the edge of the disk, and especially for features near the disk edge in the northern hemisphere (which is tilted away from us), a small error in location of the feature can change the airmass significantly. For this reason, after determining the airmass of the northern features by deprojection, we also studied the effect of varying the airmass on the model fits. For the bright northern feature observed on August 3, 2002, the best fit altitude for a two-way airmass of 8 (which is obtained from the image deprojection on a latitude-longitude grid) is 0.044 . Fits almost as good can be obtained for a two-way airmass of 6 or 10, which would place the cloud altitude at 0.048 or 0.040 bar, respectively.

The effect of underestimating the airmass is to estimate an altitude that is too deep; the real altitude is higher. Overestimating the airmass gives an altitude that is too high. Due to the rapid increase in airmass for the northern hemisphere, the first type of error would be more likely to occur because of imprecise location of the feature. Our studies of the effect of varying airmass indicate that the northern features are truly at high altitudes and this is not an artifact of errors in determining the airmass. We further found that the best fit airmasses for northern hemisphere features did indeed coincide with the locations determined from the deprojections. 


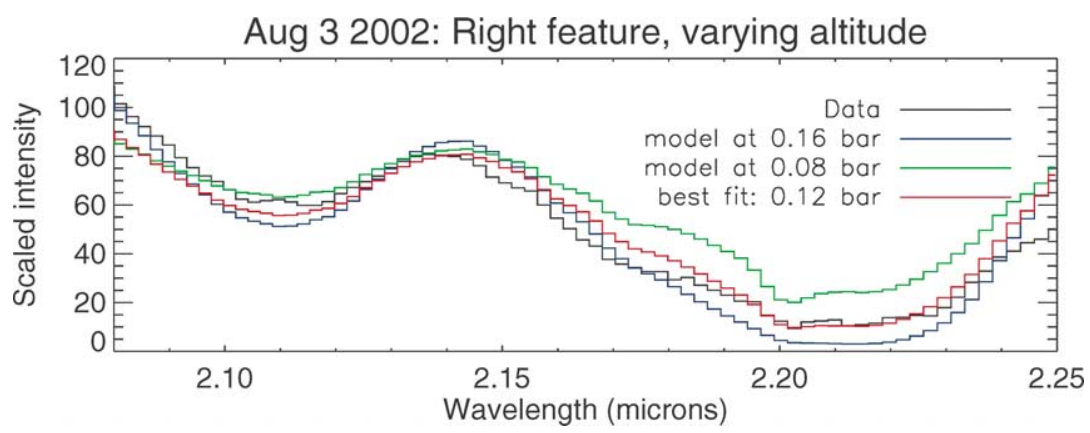

Fig. 8. The effect of varying the model altitude (pressure level) from the best fit value for the right feature from the August 3 , 2002, 12:34, data. The best fit is at 0.12 bar; models with $P=0.08$ or $P=0.16$ bar are shown for comparison.

Aug 3 2002: Right feature, varying fhe

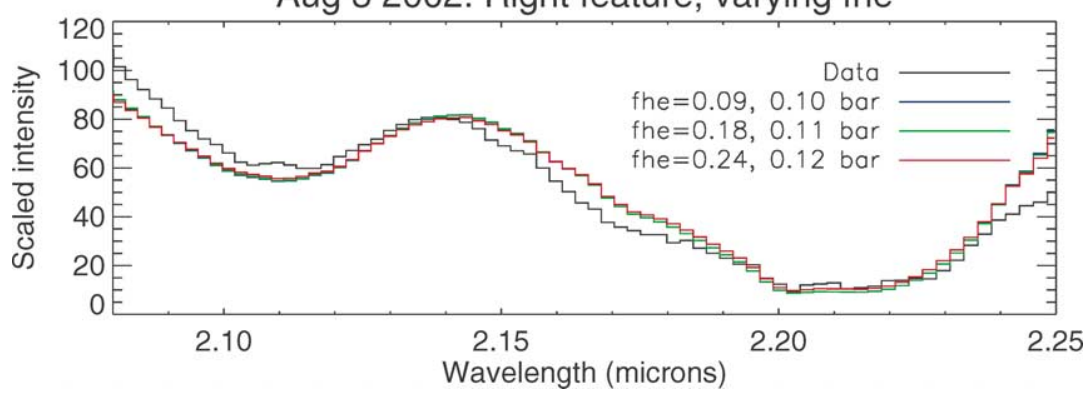

Fig. 9. The effect of varying $\mathrm{f}_{\mathrm{He}}$, shown in this case for the right feature from the August 3, 2002, 12:34, data. The goodness of the fit is not very sensitive to this parameter, but the best fit is found for a value of $\mathrm{f}_{\mathrm{He}}=0.24$ and an altitude of 0.12 bar. Fits at lower $\mathrm{f}_{\mathrm{He}}(0.09$ and 0.18 are shown) give a best fit altitude of 0.10 bar and 0.11 bar, respectively.

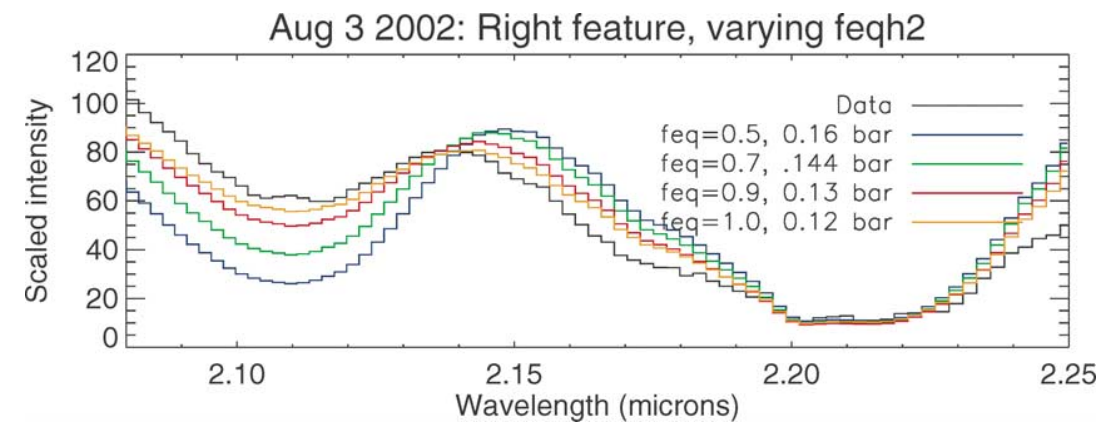

Fig. 10. The effect of varying $\mathrm{f}_{\mathrm{eq}}$, shown in this case for the right feature from the August $3,2002,12: 34$, data. The best fit value of $\mathrm{f}_{\mathrm{eq}}=1.0$ gives a feature altitude of 0.12 bar. Varying $f_{\text {eq }}$ has a strong impact on the goodness of the fit; a value of $f_{\text {eq }}=0.5$ is a poor fit to the data and the best fit requires a feature altitude of 0.16 bar. A value of $\mathrm{f}_{\mathrm{eq}}=0.9$ provides a somewhat better, but still inferior fit with a best fit altitude of 0.13 bar.

\section{Discussion}

\subsection{Sources of error}

In order to estimate the accuracy of our analysis we must consider several possible sources of error. As mentioned above, there are errors in the airmass of features located near the disk edges or in the northern hemisphere. We estimate, based on the modeling of northern features at varying airmasses discussed above, that these errors may lead to a $\pm 10 \%$ error in feature altitude in the most extreme cases. Another possible source of error is the fact that we model the bright features as flat layers at a single altitude. If the feature instead extends down through several layers (with an opti- cal depth $\leqslant 1$ in the topmost layer), then this would also be inconsistent with our simplified modeling. It is known that the stratospheric haze on Neptune does not consist of a single layer, but is instead located in several haze layers (Baines et al., 1995a). Since our modeling ignores the 'background' haze layers this may affect the accuracy of the modeling. In particular there is concern about residual light that may affect the shape of the spectrum in the $\mathrm{H}_{2}$ absorption region. In order to gauge the effect of this residual light we have examined a region of Neptune that appears to be free of bright features (the equatorial region) to gauge the background residual light. Figure 11 shows an example of the spectrum of the equator compared to that of the bright features. The equatorial spectrum is nearly flat (although there 


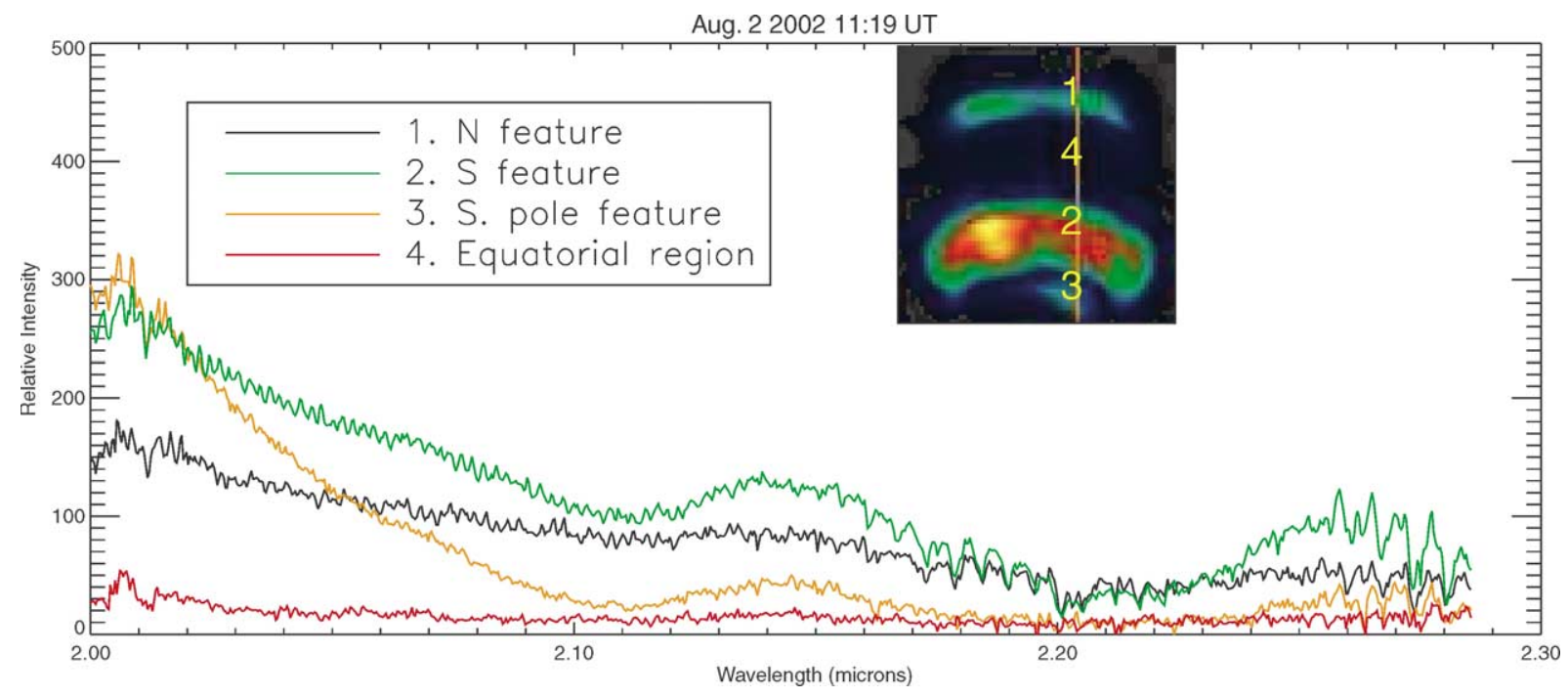

Fig. 11. A comparison of the dark equatorial region to the bright features of the planet. The spectrum of the equatorial region is nearly flat, with a flux at most $10-20 \%$ of that of the bright features. This illustrates that a uniform, background haze has only a small effect on the shape of the spectrum even in the 2.1-2.2 micron region.

is a slightly increased flux in the $2.1-2.2 \mu$ region) and the amount of residual light in the $2.1-2.2 \mu$ region is less than $10 \%$ of that of the brighter features, and less than $20 \%$ of that of the darker (south polar) feature. For this reason we feel that the residual light does not have a major effect on the accuracy of our modeling.

In some cases (for example, the data from July 18, 2000), a relatively poor fit in the $2.10-2.15$ micron range could be due to the presence of cloud layers at different altitudes, or an optically-thin cloud layer with a significant vertical extent. Attempting to reproduce such extended features would require a more sophisticated model that includes multiple scattering between cloud layers. Such a model is beyond the scope of this paper. We have also assumed that the cloud properties do not vary as a function of wavelength, which seems reasonable for the narrow wavelength range considered here. Another question we might consider is whether the features we observe are resolved or unresolved; that is, whether features cover the entire area of the pixel or only a fraction of it. Some of the features we have observed are indeed resolved (such as the longitudinal slice taken on $\mathrm{Au}-$ gust 3,2002), while others are not. What this means is that the light we receive from unresolved features is a combination of the bright feature and its surroundings. Since the surroundings have very low signal (Fig. 11), for unresolved features we have effectively a dilution of the signal from the bright feature. If we were attempting to calculate absolute I/F values (as Sromovsky et al., 2001c, did), this would be important, but this does not make any difference in our determination of cloud heights, which depends only on the relative fluxes at different wavelengths.

A second source of error comes from the model itself. There are errors associated with the uncertainty in Neptune's pressure-temperature structure (Lindal, 1992), and assumption of a constant $P-T$ profile at different latitudes on the planet. The methane $k$-coefficients used are extrapolated from Irwin et al. (1996) laboratory measurements at much warmer temperatures than those relevant to Neptune. It is difficult to know how this would bias the results; however our results are largely independent of Neptune's methane concentration in stratosphere or troposphere, which indicates that our uncertainly in the methane coefficients in not a major limiting factor in our accuracy. The fitting is indeed dominated by the $\mathrm{H}_{2}$ collision-induced absorption, which is well known at these temperatures.

Our studies of the variation of best-fit altitude with changes in the parameter $\mathrm{f}_{\mathrm{He}}$ indicates that we cannot constrain this variable to any great extent; while the best fit values are consistently $\mathrm{f}_{\mathrm{He}}=0.24$, smaller values also give a reasonable fit at a somewhat lower altitude. The effect of changing $\mathrm{f}_{\mathrm{He}}$ to 0.09 is a raising in altitude by about $20 \%$, or about $10 \%$ for $\mathrm{f}_{\mathrm{He}}=0.18$. Conrath et al. (1993) found a value of $\mathrm{f}_{\mathrm{He}}=0.15$, which is not inconsistent with our data. In the case of $f_{e q}$, the best fit is for $f_{\text {eq }}=1.0$, with reasonable model fits requiring $\mathrm{f}_{\mathrm{eq}} \geqslant 0.8$. Baines et al. (1995b) found an equilibrium ortho/para ratio to be the best fit for Neptune, and Baines and Smith (1990) also found $\mathrm{f}_{\mathrm{eq}}=1.0$, with a lower limit of 0.85 . A reanalysis of Voyager IRIS data by Conrath et al. (1998) also indicated values of $\mathrm{f}_{\mathrm{eq}}$ that were close to equilibrium. Our results are thus consistent with previous work.

\subsection{Variation of feature altitudes with latitude}

We have investigated three main classes of features on Neptune: northern hemisphere features at $30-45^{\circ}$, southern hemisphere features in the range $-30^{\circ}--50^{\circ}$, and far southern feature(s) at $-70^{\circ}$. We have found that the northern hemisphere features are consistently at higher altitudes than the southern ones $(0.023-0.064$ vs $0.10-0.21$ bars). There 
may indeed be a trend toward increasing feature altitude from south to north (we have only two data points for the far southern feature(s) which are located at $-70^{\circ}$; the best fit altitudes are 0.17 and 0.27 bars).

Our findings are consistent with the estimation of relative cloud heights from shadows cast on a background cloud layer in Voyager imaging data. Smith et al. (1989) found a mean height of $100 \mathrm{~km}$ above the underlying cloud deck for features at 27 degrees North, and $50 \mathrm{~km}$ for features at 71 degrees South. Assuming upper tropospheric temperatures and assuming that the underlying cloud deck lies at 3 bar, they estimated that the Southern clouds were close to the tropopause. Observations by Roe et al. (2001) (southern feature altitude of 0.144 and northern feature at 0.084 ), also indicate a trend of higher altitude features in the north. The northern features we observed at 0.023 and 0.04 bars are at higher altitudes than previously observed features, while a feature at $-70^{\circ}$ and 0.27 bars is the deepest feature reported thus far. A comparison with features identified by Sromovsky et al. (2001c) shows that they estimated a pressure level of $0.06 \pm 0.02$ bars for a feature at $38^{\circ} \mathrm{N}$, $0.19 \pm 0.04$ bars for a feature at $30^{\circ} \mathrm{S}$, and $0.23 \pm 0.04$ bars for a feature at $45^{\circ} \mathrm{S}$, numbers that are consistent with pressures we find for features at similar latitudes.

The infrared-bright features discussed in this paper are bright due to sunlight reflected off clouds or haze layers. We derived the altitudes of these regions by assuming the reflecting layers can be represented by a reflecting sheet of material at a particular altitude, but we cannot determine the composition of the particulates. According to Baines and Smith (1990), the stratospheric hazes most likely are made up of hydrocarbons, and the tropospheric haze may be methane haze. We typically see the northern features at altitudes of 0.023-0.06 bars, clearly in Neptune's stratosphere. In contrast, the southernmost feature is seen much deeper, $\sim 0.2$ 0.3 bars, which puts it in the upper troposphere. The features at mid-southern latitudes are located near $0.1-0.15$ bar level, near the tropopause. Moses et al. (1992) have considered the critical level for the onset of nucleation for constituents in Neptune's atmosphere. The onset for methane nucleation is $1 \mathrm{bar}$ and for photolytic products ranges from 0.0010.060 bar. Thus bright features located at pressures greater than 0.1 bar are likely methane ice clouds which are formed in the troposphere and subsequently entrained by convective upwellings to higher altitudes (Baines et al., 1995a), whereas features at $0.023-0.06$ bar are likely the result of photolytic haze enhancement.

The altitude of tropospheric methane clouds is important to our understanding of the chemistry and dynamics of Neptune. Photochemical haze production cycles depend on the delivery of methane across the cold trap to the stratosphere. Methane cloud entrainment may play a significant role in methane transport, along with diffusion of methane from the troposphere. Our results indicate that convection is an efficient means of delivering methane to the tropopause in the Southern midlatitudes $\left(-30^{\circ}--50^{\circ}\right)$ where clouds reach as high as 0.1 bar at the bottom of the stratosphere. In the South polar feature at $-70^{\circ}$ where clouds reach as high as $0.17 \mathrm{bar}$, methane clouds perhaps do not reach the tropopause but may increase methane concentration for diffusive transport to the stratosphere. The existence of higher altitude methane clouds at southern midlatitudes is in agreement with the meridional motions determined from Voyager data. Conrath et al. (1989) analyzed $200-450 \mathrm{~cm}^{-1}$ IRIS data and applied a radiative dynamical model to deduce a global circulation pattern of upwelling at southern midlatitudes and subsidence at low and high latitudes in the troposphere. The entrainment of the South polar $\left(-70^{\circ}\right)$ feature is likely related to local dynamical activity rather than meridional circulation.

The Northern haze enhancement features in the stratosphere are perhaps linked to local dynamical features. These are at the same latitude as the companion cloud to the Northern dark spot NGDS-32 (Hammel et al., 1995; Sromovsky et al., 2002). These also could be precipitated by a down draft in the stratosphere. Bézard et al. (1991) have inferred from Voyager IRIS $729 \mathrm{~cm}^{-1}$ data subsidence at 30 degrees latitude, however their data does not extend north of 30 degrees.

\section{Conclusions}

Spectroscopy of bright features on Neptune in the 2micron range is a good diagnostic of the features' vertical location because of the spectrum's sensitivity to the hydrogen absorption in this wavelength interval. Using adaptive optics to obtain a spatial resolution of $0.06^{\prime \prime}$ (which is 40 resolution elements across the disk or about $1200 \mathrm{~km}$ on Neptune), we have estimated the best-fit altitudes of 18 bright features seen at various latitudes on Neptune in 2000 and 2002. Our results agree with previous observations that Neptune's bright cloud features are located at higher altitudes in the northern hemisphere and deeper in the south; however with the use of adaptive optics we are able to clearly identify individual features and thus to make a more accurate determination of cloud height as a function of latitude.

We find that in the bright bands extending from $-30^{\circ}$ to $-50^{\circ}$ in Neptune's southern hemisphere most of the features are located at altitudes of $0.10-0.14$ bars. A small feature observed in both 2000 and 2002 at $-70^{\circ}$ appears to be located at deeper in the atmosphere at $0.17-0.27$ bars. Features in the northern hemisphere are uniformly at higher altitudes, ranging from 0.023-0.064 bars, which is above the tropopause. Comparisons to suggested circulation patterns on Neptune indicates that the southern mid-latitude features are methane haze circulated up from below, while the southernmost feature may be an isolated area of upwelling in a general area of subsidence. Northern bright features, with altitudes in the stratosphere, may be due to subsidence of stratospheric haze material rather than upwelling and condensation of methane gas. 


\section{Acknowledgments}

Our thanks to referees Thierry Fouchet and Kevin Baines, who provided valuable suggestions for the improvement of this paper. This work has been supported in part by the National Science Foundation Science and Technology Center for Adaptive Optics, managed by the University of California at Santa Cruz under cooperative agreement No. AST 9876783. This work was performed under the auspices of the US Department of Energy, National Nuclear Security Administration, by the University of California, Lawrence Livermore National Laboratory under contract No. W-7405Eng-48. Data presented herein were obtained at the W.M. Keck Observatory, which is operated as a scientific partnership among the California Institute of Technology, the University of California and the National Aeronautics and Space Administration. The Observatory was made possible by the generous financial support of the W.M. Keck Foundation.

\section{References}

Baines, K.H., Hammel, H.B., 1994. Clouds, hazes, and the stratospheric methane abundance in Neptune. Icarus 109, 20-39.

Baines, K.H., Smith, H.W., 1990. The atmospheric structure and dynamical properties of Neptune derived from ground-based and IUE spectrophotometry. Icarus $85,65-108$.

Baines, K.H., Hammel, H.B., Rages, K.A., Romani, P.N., Samuelson, R.E., 1995a. Clouds and hazes in the atmosphere of Neptune. In: Cruikshank, D.P. (Ed.), Neptune and Triton. Univ. of Arizona Press, Tucson, pp. 489-546.

Baines, K.H., Mickelson, M.E., Larson, L.E., Ferguson, D.W., 1995b. The abundances of methane and ortho/para hydrogen in Uranus and Neptune: implications of new laboratory $4-0 \mathrm{H}_{2}$ quadrupole line parameters. Icarus 114, 328-340.

Bézard, B., Romani, P.N., Conrath, B.J., Maguire, W.C., 1991. Hydrocarbons in Neptune's stratosphere from Voyager infrared observations. J. Geophys. Res. 96, 18961-18975.

Borysow, A., 1991. Modeling of collision-induced infrared absorption spectra of $\mathrm{H}_{2}-\mathrm{H}_{2}$ pairs in the fundamental band at temperatures from 20 to $300 \mathrm{~K}$. Icarus 92, 273-279.

Borysow, A., Frommhold, L., 1989. Collision-induced infrared spectra of $\mathrm{H}_{2}-\mathrm{He}$ pairs at temperatures from 18 to $7000 \mathrm{~K}$. II. Overtone and hot bands. Astrophys. J. 341, 549-555.

Borysow, A., Frommhold, L., Moraldi, M., 1989. Collision-induced infrared spectra of $\mathrm{H}_{2}-\mathrm{He}$ pairs involving $0-1$ vibrational transitions and temperatures from 18 to $7000 \mathrm{~K}$. Astrophys. J. 336, 495-503.

Colina, L., Bohlin, R.C., Castelli, F., 1996a. The 0.12-2.5 micron absolute flux distribution of the Sun for comparison with solar analog stars. Astron. J. 112, 307-315.

Colina, L., Bohlin, R.C., Castelli, F., 1996b. Absolute flux calibrated spectrum of Vega. Instrum. Sci. Rep. OSG-CAL-96-01, Baltimore: STSCI.

Conrath, B.J., Flasar, F.M., Hanel, R., Kunde, V., Maguire, W., Pearl, J., Pirraglia, J., Samuelson, R., Gierasch, P., Weir, A., Bezard, B., Gautier, D., Cruikshank, D., Horn, L., Springer, R., Shaffer, W., 1989. Infrared observations of the neptunian system. Science 246, 1454-1459.

Conrath, B.J., Gautier, D., Owen, T.C., Samuelson, R.E., 1993. Constraints on $\mathrm{N}_{2}$ Neptune's atmosphere from Voyager measurements. Icarus 101, 168-171.

Conrath, B.J., Gierasch, P.J., Ustinov, E.A., 1998. Thermal structure and para hydrogen fraction on the outer planets from Voyager IRIS measurements. Icarus 135, 501-517.

Gibbard, S.G., de Pater, I., Roe, H., Macintosh, B., Gavel, D., Max, C.E., Baines, K.H., Ghez, A., 2002. High-resolution infrared imaging of Neptune from the Keck Telescope. Icarus 156, 1-15.
Goody, R.M., Yung, Y.L., 1989. Atmospheric Radiation. Oxford Univ. Press, New York.

Hammel, H.B., 12 colleagues, 1989. Neptune's wind speeds obtained by tracking clouds in Voyager images. Science 245, 1367-1369.

Hammel, H.B., Lockwood, G.W., Mills, J.R., Barnet, C.D., 1995. Hubble Space Telescope imaging of Neptune's cloud structure in 1994. Science 268, 1740-1742.

Irwin, P.G.J., Calcutt, S.B., Taylor, F.W., Weir, A.L., 1996. Calculated $k$ distribution coefficients for hydrogen- and self-broadened methane in the range $2000-9500 \mathrm{~cm}^{-1}$ from exponential sum fitting to band-modelled spectra. J. Geophys. Res. 101, 26137-26154.

Lacis, A.A., Oinas, V., 1991. A description of the correlated- $k$ distribution method for modelling nongray gaseous absorption, thermal emission, and multiple scattering in vertically inhomogeneous atmospheres. J. Geophys. Res. 96, 2027.

Lindal, G.F., 1992. The atmosphere of Neptune: an analysis of radio occultation data acquired with Voyager 2. Astron. J. 103, 967-982.

Martin, S.C., Roe, H., de Pater, I., Macintosh, B., Gibbard, S., Max, C.E., Gavel, D., Brown, M., Ghez, A., 2000. The evolution of cloud features on Neptune over a 20-day time period. AAS DPS meeting \#32, \#10.17.

Martin, S.C., de Pater, I., Roe, H., Macintosh, B.A., Gibbard, S., Max, C.E., 2001. The morphology and motions of storm features on Neptune on minute and hour timescales. AAS DPS meeting \#33, \#22.05.

Martin, S.C., de Pater, I., Gibbard, S.G., Macintosh, B.A., Roe, H.G., Max, C.E., 2002. The motions and morphologies of cloud features on Neptune: continued monitoring with Keck adaptive optics. AAS DPS meeting \#34, \#13.12.

Max, C.E., Macintosh, B.A., Gibbard, S., Gavel, D.T., Roe, H., de Pater, I., Ghez, A.M., Acton, S., Wizinowich, P.L., Lai, O., 2000. Neptune and Titan observed with Keck Telescope adaptive optics. In: Proc. SPIE, Vol. 4007, p. 803-810.

Max, C.E., Macintosh, B.A., Gibbard, S.G., Gavel, D.T., Roe, H.G., de Pater, I., Ghez, A.M., Acton, D.S., Lai, O., Stomski, P., Wizinowich, P.L., 2002. Cloud structures on Neptune observed with Keck Telescope adaptive optics. Astron. J. 125, 364-375.

McClean, I., Graham, J.R., Becklin, E.E., Figer, D.F., Larkin, J.E., Levenson, N.A., Teplitz, H.I., 2000. Performance and results with the NIRSPEC echelle spectrograph on the Keck II Telescope. In: Masanori, I., Moorwood, A.F. (Eds.), Optical and IR Telescope Instrumentation and Detectors. In: Proc. SPIE, Vol. 4008, pp. 1048-1055.

Moses, J.I., Allen, M., Yung, Y.L., 1992. Hydrocarbon nucleation and aerosol formation in Neptune's atmosphere. Icarus 99, 318-346.

Roddier, F., Roddier, C., Graves, J.E., Northcott, M.J., Owen, T., 1998. NOTE: Neptune's cloud structure and activity: ground-based monitoring with adaptive optics. Icarus 136, 168.

Roe, H.G., Graham, J.R., McLean, I.S., de Pater, I., Becklin, E.E., Figer, D.F., Gilbert, A.M., Larkin, J.E., Levenson, N.A., Teplitz, H.I., Wilcox, M.K., 2001. The altitude of an infrared-bright cloud feature on Neptune from near-infrared spectroscopy. Astron. J. 122, 1023-1029.

Romani, P.N., Atreya, S.K., 1988. Methane photochemistry and methane production on Neptune. Icarus 74, 424-445.

Rousselot, P., Lidman, C., Cuby, J.-G., Moreels, G., Monnet, G., 2000. Night-sky spectral atlas of $\mathrm{OH}$ emission lines in the near-infrared. Astron. Astrophys. 354, 1134-1150.

Smith, B.A., 64 colleagues, 1989. Voyager 2 at Neptune: imaging science results. Science 246, 1422-1449.

Sromovsky, L.A., Limaye, S.S., Fry, P.M., 1993. Dynamics of Neptune's major cloud features. Icarus 105, 110-141.

Sromovsky, L.A., Limaye, S.S., Fry, P.M., 1995. Clouds and circulation on Neptune: implications of 1991 HST observations. Icarus 118, 25-38.

Sromovsky, L.A., Fry, P.M., Baines, K.H., Limaye, S.S., Orton, G.S., Dowling, T.E., 2001a. Coordinated 1996 HST and IRTF imaging of Neptune and Triton. I. Observations, navigation, and differential deconvolution. Icarus 149, 416-434.

Sromovsky, L.A., Fry, P.M., Baines, K.H., Dowling, T.E., 2001b. Coordinated 1996 HST and IRTF imaging of Neptune and Triton. II. Implications of disk-integrated photometry. Icarus 149, 435-458. 
Sromovsky, L.A., Fry, P.M., Dowling, T.E., Baines, K.H., Limaye, S.S., 2001c. Coordinated 1996 HST and IRTF imaging of Neptune and Triton. III. Neptune's atmospheric circulation and cloud structure. Icarus 149, 459-488.

Sromovsky, L.A., Frye, P.M., Baines, K.H., 2002. The unusual dynamics of northern dark spots on Neptune. Icarus 156, 16-36.
Stratman, P.W., Showman, A.P., Dowling, T.E., Sromovsky, L.A., 2001. EPIC simulations of bright companions to Neptune's great dark spots. Icarus 151, 275-285.

Zheng, C., Borysow, A., 1995. Modeling of collision-induced infrared absorption spectra of $\mathrm{H}_{2}$ pairs in the first overtone band at temperatures from 20 to $500 \mathrm{~K}$. Icarus $113,84-90$. 